\title{
La Formación Universitaria del Auditor para Prevenir y Detectar el Fraude Contable en la Empresa
}

\section{University Training of Auditor to Prevent and Detect Fraud in Business Accounting}

' Alexis EfraínAlpaca Cusicanqui

\section{RESUMEN:}

Esta investigación se ha efectuado para conocer cuál es el grado de preparación de los alumnos de la Facultad de Ciencias Contables y Financieras de la Universidad Nacional Jorge Basadre Grohmann, sobre un tema que está afectando a muchas empresas: El fraude contable. Los resultados principales que se encontraron en la investigación fueron que los alumnos están recibiendo una preparación competente respecto al desarrollo de distintos tipos de Auditoria, pero que a su vez no conocen mucho acerca del riesgo del frande. De igual manera, se establece que los docentes están haciendo su esfuerzo por enseñar las asignaturas de auditoria, éstas no contienen mucha información respecto del fraude, del control interno con enfoque a los riesgos y de la gestión de riesgos empresariales.

Palabras clave: Formación profesional, riesgo, control interno, fraude contable, auditoría, gestión de riesgos empresariales.

\section{ABSTRACT:}

This investigation has been applied to know which is the preparation degree of the students of the Accounting and Financial Sciences Faculty of Jorge Basadre Grohmann National University, it is a topic that is concerning many companies: The countable fraud. The principal results found in the investigation were that the students are receiving a competent preparation about the development of different types of Audit, but at the sume time, they do not know much about the fraud risk. Any way, it is stablished that the professors are doing his effort for teaching the subjects of Audit. they do not contain much information ahout the fraud, the internal control with approach to the risks, and the management of managerial risks.

Keywords: Professional training, risk, internal control, counlable frand, audit, enterprise risks management.

\footnotetext{
' Master Sciencie con mención en Contabilidad: Aaditoria, Contador Público. Facultad de Ciencias Juridicas y Empresariales. Universidad Nacional Jorge Basadre Grohmann
} 


\section{INTRODUCCIÓN}

Las actividades fraudulentas son más frecuentes en lugares de trabajo con poca o ninguna supervisión, politicas y procedimientos inadecuados, y controles y recursos preventivos insuficientes. El fraude a menudo ocurre en los niveles más altos de la empresa, incluso estafadores experimentados aprovechan la protección que les brindan estas circunstancias y siguen operando durante años sin ser detectados. Las repercusiones del fraude no se limitan al nivel interno. Las exigencias y los controles que imponen reguladores, grupos de interés y aseguradoras, entre otros, tanto en el ámbito local como en el internacional hacen que directores y accionistas sean personalmente responsables por las fallas que puedan existir en su organización.

Las evaluaciones de riesgo de carácter periódico reducen las pérdidas y mejoran la eficacia organizacional beneficios que cubren sus costos en un factor de diez a uno. Una investigación rápida y profunda de las personas, las transacciones y los activos bajo sospecha también puede recuperar las pérdidas, evitar futuras pérdidas y revelar la identidad de los posibles infractores.

La formación universitaria está definida como el "conjunto de actividades destinadas a proporcionar los conocimientos, habilidades y destrezas que permitan desarrollar capacidades y valores para ejercer una ocupación o diversas funciones con competencia y eficacia profesional".

La formación profesional se extiende a través de un conjunto complejo de procesos y acciones que incluyen: la relación profesor - alumno, la infraestructura educativa. El curriculo de estudios, el apoyo administrativo, los equipos tecnológicos, incluso la situación social y psicológica del alumno.

Parte de esta formación profesional del estudiante de Contabilidad corresponde a las asignaturas de Auditoría las cuales servirán para formar al futuro Contador con los conocimientos suficientes para desempeñarse como auditor. En el desempeño profesional, sea como contador o como auditor es indispensable conocer los temas relacionados al fraude en general y de manera especifica al fraude Contable. En cualquier caso, en situaciones de este tipo, es importante que el auditor entienda la naturaleza del fraude, las causas que to originan y las técnicas que deben utilizarse una vez que el fraude ya se ha producido. El auditor, tal y como indica la Guia Internacional de Auditoría No 11 (IFAC, 1982). "al formar una opinión sobre los estados financieros ... cualquier indicio en el sentido de que. ha podido haber algún tipo de fraude o error al respecto. hará que el auditor extienda sus procedimientos para confirmar o rechazar tales suposiciones" (pág, 1). Por todo ello. es conveniente y aconsejable que el auditor pueda desarrollar un papel activo en situaciones en las que existe información financiera fraudulenta. El reconocimiento de la capacidad del auditor, ante estas situaciones, es un hecho sumamente importante que debe quedar establecido como premisa básica, antes de entrar en el terreno de asignación de responsabilidades.

\section{Objetivo General}

Precisar los conocimientos que deben adquirir los alumnos de la Facultad de Ciencias Contables y Financieras, para detectary prevenir el fraude contable.

\section{MATERIALES Y MÉTODOS}

\subsection{Tipo de investigación}

El tipo de Investigación es de diseño No Experimental, porque se ha observado hechos o situaciones tal como se dan en su contexto natural para después analizarlos. De acuerdo a su finalidad será aplicada. Según su profundidad será descriptiva y explicativa.

\subsection{Población y muestra del estudio}

La población está conformada por estudiantes universitarios, de ambos sexos, del quinto año de la Facultad de Ciencias Contables y Financieras de la Universidad Nacional Jorge Basadre Grohmann. Debido a que la población es pequeña, no fue necesario establecer una muestra por lo que la encuesta se aplicó a toda la población.

\subsection{Hipótesis y variables}

\section{Hipólesis}

"La formación profesional de los alumnos que egresan de la Facultad de Ciencias Contables y Financieras de la Universidad Nacional Jorge Basadre Grohmann, los capacita para detectar y prevenir el fraude contable"

\section{Variables}

Variable independiente: Formación profesional Variable Dependiente: Detección y prevención del Fraude Contable

\subsection{Técnicas de Recolección de Datos}

Para poder obtener datos significativos fue necesario elaborar un cuestionario de preguntas para aplicarlo a través de una encuesta a la población de alumnos. EI cuestionario aplicado consta de preguntas cerradis dicotómicas y de múltiples respuestas.

\section{RESULTADOS}

Con la finalidad de conocer la opinión de los alumnos sobre los contenidos de las asignaturas que corresponden al área de Auditoría, se efectuó la siguiente pregunta, ¿Cree usted que los contenidos de las asignaturas de Auditoría que se dictan en la FACF son óptimos? Obteniendo los siguientes resultados:

Tabla $\mathrm{N}^{\circ} 01$. Contenidos de las asignaturas de Auditoría de la FACF son óptimos

\begin{tabular}{ccc}
\hline Respuestas & Frecuencia & Porcentaje \\
\hline $\mathrm{Si}$ & 45 & $73 \%$ \\
No & 17 & $27 \%$ \\
Total & 62 & $100 \%$ \\
\hline
\end{tabular}

Fuente: Encuesta elaborada por el autor 


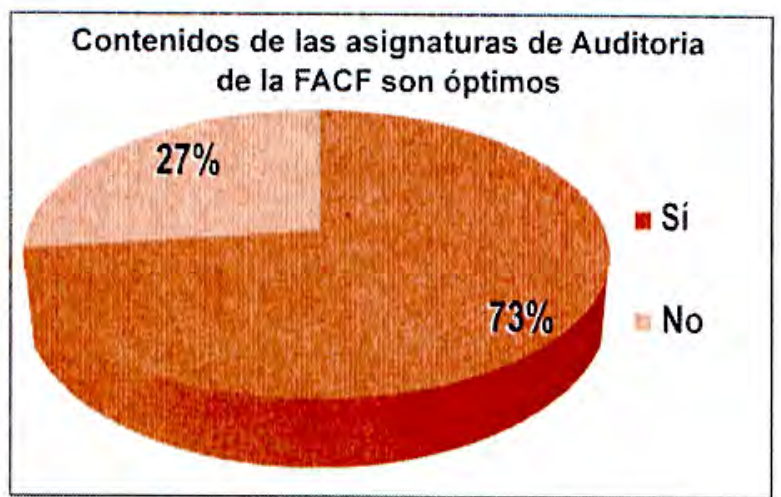

Figura $\mathrm{N}^{\circ} \mathbf{0 1}$. Contenidos de las asignaturas de Auditoría de la FACF son óptimos

Fuente: Encuesta elaborada por el autor

Del total de los alumnos encuestados, el $73 \%$. manifiesta que los contenidos de las asignaturas relacionadas a la Auditoria que se dictan en la Facultad de Ciencias Contables y Financieras, podrian mejorarse para permitir obtener mejores capacidades y destrezas necesarias para desempeñarse en el campo de la Auditoria; mientras que un $27 \%$ de los encuestados considera que los contenidos de las asignaturas son óptimos y permiten obtener el conocimiento adecuado sobre auditoria.

Con la finalidad de conocer si en las asignaturas de Auditoria que se dictan en la FACF, se imparte a los alumnos información sobre el fraude Contable, se efectuó la pregunta, ¿En las asignaturas de Auditoria ha recibido conocimientos sobre el fraude Contable? Obteniendo los siguientes resultados:

Tabla $\mathbf{N}^{\circ} 02$. Conocimientos recibidos sobre el fraude Contable

\begin{tabular}{ccc}
\hline Respuestas & Frecuencia & Porcentaje \\
\hline $\mathrm{Si}$ & 15 & $24 \%$ \\
$\mathrm{No}$ & 47 & $76 \%$ \\
Total & 62 & $100 \%$ \\
\hline
\end{tabular}

Fuente: Encuesta elaborada por el autor

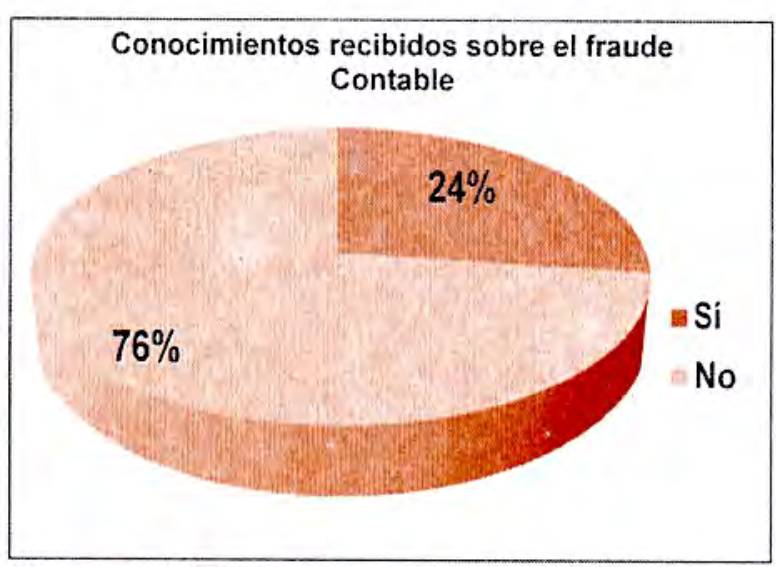

Figura $\mathrm{N}^{\circ} \mathbf{0 2}$. Conocimientos recibidos sobre el fraude Contable

Fuente: Encuesta elaborado por el autor
Del total de los alumnos encuestados, el $76 \%$, manifiesta que en las asignaturas relacionadas a la Auditoria que se dictan en la Facultad de Ciencias Contables y Financieras, no han recibido correcta información acerca del fraude contable; mientras que un $24 \%$ de los encuestados considera que si ha recibido información correspondiente a este tema.

\section{DISCUSIÓN}

De acuerdo a los resultados obtenidos, se puede expresar que los alumınos están recibiendo una gran cantidad de conocimientos para su formación y desempeño profesional como auditores. Asimismo, los docentes están haciendo grandes esfuerzos por transmitir los conocimientos, utilizando técnicas de enseñanza que en algunos casos podría mejorarse para lograr los objetivos perseguidos.

Sin embargo, debe precisarse que en los contenidos de las asignaturas de Auditoría, muy poco se considera el tema de la gestión de riesgos empresariales que involucra el riesgo de fraude, por lo que el alumno que egresa de las aulas de la FACF está altamente capacitado para trabajar en el campo de la auditoría, pero específicamente conoce poco acerca del riesgo de fraude contable, el cual está intimamente relacionado con la implementación, ejecución y evaluación del control interno con enfoque en riesgos. Es importante que el profesional contable sea un amplio conocedor de este flagelo que afecta gravemente a las empresas. En este sentido los alumnos de la Facultad de Ciencias Contables y Financieras necesitan que se les amplie las enseñanzas relacionadas a este tema, el cual se presenta a menudo en el desarrollo de sus trabajos, no sólo de auditoria, sino también al desempeñarse como Contadores.

\section{V.CONCLUSIONES}

En la actualidad el fraude contable es un tema que se estudia con énfasis en todo el mundo, debido a que se presenta en muchas empresas de diversas maneras, ocasionando graves perjuicios a la empresa y a la sociedad.

Los estudiantes de la Facultad de Ciencias Contables y Financieras egresan de las aulas universitarias con amplios conocimientos sobre Auditoria como resultado de haber estudiado siete asignaturas correspondientes a esta área. Sin embargo, las asignaturas del área de Auditoria, tienen en su contenido escasos temas relacionados al fraude contable y al inminente riesgo que existe de que éste se desarrolle en las empresas.

\section{RECOMENDACIONES}

Es importante que todos los estudiantes y profesionales de la carrera Contable, tomen en cuenta un estudio e investigación a conciencia sobre el fraude contable, que hoy en día, involucra en actos de corrupción tanto a empleados como a funcionarios de las empresas, desde el más bajo nivel hasta el más alto; con la finnalidad de poder minimizar los riesgos de su desarrollo dentro de 
las empresas.

Es conveniente, poder incrementar una asignatura en al cual se pueda presentar al estudiante de Contabilidad. todos los aspectos relacionados al Control interno con enfoque en riesgos, entre los que se encuentra el riesgo de fraude, desarrollando en esta nueva asignatura ítems importantes de los documentos que se han venido presentando en el mundo para ayudar a prevenir y detectar los riesgos de fraude en las empresas, tales como el informe $\mathrm{COSO} 2$, entre otros.

\section{REFERENCIAS BIBLIOGRÁFICAS}

Arroyo Morales Angélica, 1997. Metodología de la Investigación Científica, Cusco - Perú, Facultad de Ciencias Contables y Financieras - UNSAAC, Segunda Edición

Barrón Tirado Concepción, Rojas Moreno Ileana, Sandoval Montaño Rosa. Tendencias en la formación profesional universitaria en educación. Revista Perfiles Educativos, enero-marzo, número 71. Universidad Nacional Autónoma de México. México D. F. 1996

Comer Michael J. El Fraude en la Empresa. Ediciones Deusto SA. Segunda edición. España. 1984.

Federación Internacional de Contadores - IFAC. Manual Internacional de Pronunciamientos de Auditoría y Aseguramiento. Fondo Editorial del Colegio de Contadores Públicos de Lima. 2004.
Garcia Benau Maria Antonia, Humphrey Christopher. La auditoria y el fraude: algunas consideraciones internacionales. Revista Espariola de Financiación y Contabilidad. Vól. XXIV, N84.Abril-junio 1995.

Hidalgo Ortega Jesús, La Tesis, 1991, Lima - Perú, Ediciones Fecat, Segunda Edición

Hidalgo Ortega, Jesús, Auditoria de Estados Financieros. Ediciones Ojeda. Lima-Perú. 1986.

Pardinas, Felipe, Metodología y Técnicas de Investigación Científica. México Siglo XXI 1985.

Sánchez Sánchez, Rohel. Las Sociedades de Auditoría ante el riesgo de fraude en los estados financieros y el nuevo contexto económico. Cuadernos de investigación de la Universidad San Pablo. Impresiones Andrea. Arequipa-Perú. 2009.

\section{Correspondencia:}

Alexis Efrain Alpaca Valeriano

Ciudad Universitaria Fundo "Los Granados"

Av. Miraflores s/n. Tacna. Perú

acapla@hotmail.com 